\title{
Wnt/ $\beta$-Catenin Pathway Balances Scaffold Degradation and Bone Formation in Tissue-Engineered Laminae
}

\author{
Hailong Li, ${ }^{1}$ Linli Li $\mathbb{D}^{1},{ }^{1}$ Yiqun He, ${ }^{1}$ Wei Mao, ${ }^{1}$ Haofei Ni, ${ }^{1}$ Aolei Yang, ${ }^{1}$ Feizhou Lyu $\mathbb{D}^{1,2}$ \\ and Youhai Dong ${ }^{1}{ }^{1}$ \\ ${ }^{1}$ Department of Orthopedics, Shanghai Fifth People's Hospital, Fudan University, China \\ ${ }^{2}$ Department of Orthopedics, Huashan Hospital, Fudan University, China \\ Correspondence should be addressed to Feizhou Lyu; lufeizhou@hotmail.com and Youhai Dong; youhaidong1964@163.com
}

Received 17 July 2021; Accepted 23 August 2021; Published 13 September 2021

Academic Editor: Jun Liu

Copyright (c) 2021 Hailong Li et al. This is an open access article distributed under the Creative Commons Attribution License, which permits unrestricted use, distribution, and reproduction in any medium, provided the original work is properly cited.

\begin{abstract}
Tissue engineering provides a promising way for the regeneration of artificial vertebral laminae. Previous studies have confirmed the feasibility of reconstructing vertebral laminae via hydroxyapatite-collagen I scaffolds and mesenchymal stromal cells. However, there were no studies exploring the degradation of hydroxyapatite-collagen I scaffolds and the function of $\mathrm{Wnt} / \beta$-catenin pathway in the process. In this study, tissue-engineered laminae (TEL) were constructed by nanohydroxyapatite/collagen I scaffolds and umbilical cord Wharton's Jelly mesenchymal stromal cells (WJ-MSCs). Cell attachment was observed by scanning electron microscopy, and cell viability was confirmed by Live/Dead staining. The rat models were randomly divided into control and $\beta$-catenin inhibition groups. Vertebral lamina defect rat models were made on the fifth lumbar vertebrate, and TEL was implanted into the defect site. After 14 weeks, the newborn laminae were harvested for microcomputed tomography, histology, or transcriptional profile analysis. We found that, for the control group, the newborn lamina formation matched with the scaffold degradation and complete newborn laminae formed at the 14th week; for the $\beta$-catenin inhibition group, the scaffold degradation rate overrated the lamina formation and no complete artificial laminae were formed at the 14th week. In addition, the osteoclastic genes, such as Cathepsin K or RANKL, in the control groups were significantly lower than the $\beta$-catenin inhibition group, and the antiosteoclastic gene, OPG, in the control group was significantly higher than the $\beta$-catenin inhibition group. In conclusion, inhibition of Wnt/ $\beta$-catenin pathway led to speedy scaffold degradation and deferred artificial lamina formation. Wnt/ $\beta$-catenin pathway played a critical role in maintaining the balance between scaffold degradation and bone formation in the process of vertebral lamina reconstruction.
\end{abstract}

\section{Introduction}

Laminectomy was a routine surgical protocol for spinal diseases with spinal stenosis $[1,2]$. Postoperative epidural scar adhesion can lead to persistent back pain and difficulty for reoperation [3-6]. Tissue engineering techniques have been successfully used to reconstruct the epidural fat or vertebral laminae to avoid epidural scar adhesion in animal studies [7-11].

The common tissue engineering approach involves the use of a biocompatible scaffold, cells, and/or a combination of bioactive molecules such as growth factors and cytokines. Due to the particular structures of the spinal canal, soft biomaterials were favored for the reconstruction of vertebral laminae avoiding compression of the spinal cord after implantation [7]. Nanohydroxyapatite/collagen I scaffolds (nHE/COL) have been well characterized and commercialized for bone defect repair [12, 13]. Its excellent osteoinduction and mechanical properties make it an ideal material for the construction of tissue-engineered laminae (TEL) $[14,15]$. Previous studies $[7,16,17]$ have successfully constructed TEL with nHE/COL scaffolds and mesenchymal stromal cell (MSC) and reconstructed vertebral laminae. However, the relationship between nHE/COL scaffold degradation and lamina formation remains unclear.

Wnt/ $\beta$-catenin signaling pathway played a crucial role in bone regeneration [18]. Transcriptional profiling and spatial gene expression analysis have found a series of 
Wnt signaling molecules that are involved in the process of fracture healing [19-21]. Besides, Wnts and their antagonists also exhibit a distinct temporal expression pattern by targeting different cell lineages during the bone regeneration process [18]. Specifically, Wnts induce self-renewal and proliferation in skeletal stem/progenitor cells during the early stages of fracture healing, and once osteogenic differentiation has been triggered in these cells, Wnts then activate the differentiation cascade [18]. Moreover, Wnts can induce the expression of OPG on osteoblasts by regulating the differentiation of osteoclasts and affecting the function of osteoclasts, which also ultimately affects the bone resorption process [22]. Thus, Wnt/ $\beta$-catenin signaling pathway is involved in bone remodeling by regulating both bone resorption and bone formation processes.

In this study, we speculated that $\mathrm{Wnt} / \beta$-catenin signaling pathway might regulate the balance between scaffold degradation and bone formation and play an important role in the reconstruction of vertebral laminae. Tissueengineered laminae (TEL) were constructed using rat umbilical cord Wharton's Jelly-derived MSC and nHE/COL scaffold. The rat models were randomly divided into control and $\beta$-catenin inhibition groups. Vertebral lamina defect rat models were made, and TEL was implanted into the defect site. After 14 weeks, the newborn laminae were harvested for microcomputed tomography, histology, or transcriptional profile analysis.

\section{Methods}

2.1. Ethics Statement. All animal experiments were performed in the Animal Facility of East China Normal University and according to the protocol (Protocol number: 20150482A168) authorized by the Animal Care and Use Committee of Fudan University.

2.2. Cell Culture. Rat mesenchymal stromal cells derived from umbilical cord Wharton's Jelly were purchased from Otwo Biotech (Guangzhou, China). All cells were cultured in Dulbecco's modified Eagle's medium (DMEM; Hyclone, UT, USA) supplemented with $10 \%$ fetal bovine serum (FBS; Biological Industries, Beit HaEmek, Israel).

2.3. Construction of Tissue-Engineered Laminae (TEL). The nHE/COL scaffold was bought from the Beijing Allgens Medical Science \& Technology Co., Ltd. Under sterile condition, the nHE/COL scaffold was cut to the size of $8 \mathrm{~mm} \times$ $6 \mathrm{~mm}$. Rat MSC at passage 4-7 was trypsinized and resuspended in media at a concentration of $1 \times 10^{6} / \mathrm{ml}$. Then, $100 \mu \mathrm{l}$ cell suspensions were pipetted on one side of each scaffold, and after $30 \mathrm{~min}, 100 \mu \mathrm{l}$ cell suspensions were pipetted on the other side. All constructs were placed in culture medium before implantation.

2.4. Live/Dead Staining. Cell viability of MSC in TEL was measured with a Live/Dead assay kit (Best-Bio, Shanghai, China). Experiments were performed according to the manufacturer's instructions. The representative images were obtained using a ZEISS confocal fluorescence microscope (ZEISS, Jena, Germany).
2.5. Scanning Electron Microscope. The TEL was fixed with electron microscopy fixative solution (Severicebio, Wuhan, China), dehydrated, mounted on an aluminum stub, and sputter-coated with gold-palladium for 30 seconds. The morphology of TEL and MSC adhesion on the scaffolds was then viewed on a scanning electron microscope (Hitachi, Tokyo, Japan).

2.6. Animal Studies. The construction of vertebral lamina defect rat models was performed as previously described [17]. Briefly, spinous processes and interspinous ligaments were removed to expose the vertebral laminae, a bone defect measuring $8 \mathrm{~mm} \times 6 \mathrm{~mm}$ was created in the vertebral laminae, and then, the TEL was placed and fixed in the bone defect. For the $\beta$-catenin inhibition group, the rats were injected intraperitoneally with XAV-939 (MCE, NJ, USA) according to the standard of $4 \mathrm{mg} / \mathrm{kg}$ at the frequency of twice a week for the first two weeks and once a week. For the control group, the rats were injected intraperitoneally with the same dose of saline.

2.7. Micro-CT Examination. The target vertebrates were harvested at the $2 \mathrm{nd}, 4 \mathrm{th}, 6 \mathrm{th}, 10 \mathrm{th}$, and 14 th weeks and fixed in $4 \%(\mathrm{w} / \mathrm{v})$ paraformaldehyde. The specimens were examined using High Resolution in vivo X-ray Microtomograph System (Bruker, Bremen, Germany). The 3D model was reconstructed manually using the NRecon Reconstruction software (1.7.4.2, Bruker) and analyzed using the CTAn software (1.18.8.0, Bruker).

2.8. Histological Staining. After the micro-CT examination, the tissue specimens were decalcified with $10 \%$ ethylenediaminetetraacetic acid for 4 weeks. Tissue sections with $6 \mu \mathrm{m}$ thickness were cut on a microtome and mounted onto glass slides. The sections were processed for routine histological analysis by hematoxylin-eosin (HE), Goldner's trichrome, tartrate-resistant acid phosphatase (TRAP), and immunohistochemistry (IHC) staining.

2.9. IHC Staining. Immunohistochemistry was performed as previously described [17]. The following antibodies were used: anti-RANKL rabbit polyclonal (GB11235, Severicebio), anti-osteoprotegerin rabbit polyclonal (GB11151, Severicebio), anti-OCN rabbit polyclonal (GB11233, Severicebio), and horseradish peroxidase- (HRP-) conjugated secondary antibodies (GB23303, Severicebio). The percentage of positive staining area was analyzed by the plugins of IHC Profiler in the Image J software (Version: 1.8.0_112, NIH, USA).

2.10. Reverse Transcription PCR. Total RNA was isolated from cells or bone tissue using TRIzol Reagent (Invitrogen) according to the manufacturer's instructions. cDNA was synthesized from total RNA (500 ng) using a reverse transcription kit (Takara, Tokyo, Japan). qPCR was performed in triplicate using $1 \mu \mathrm{L}$ of cDNA in a standard SYBR premix Ex Taq (Takara) on the Applied Biosystems 7500 Real-Time PCR Detection System (Applied Biosystems, CA, USA). GAPDH served as an internal control. The following primers were used: GAPDH, 5' -GGCACAGTCAAGGCTG AGAATG-3' and $5^{\prime}$-ATGGTGGTGAAGACGCCAGTA- 
$3^{\prime}$; RANKL, $5^{\prime}$-ATGATGGAAGGTTCGTGG- ${ }^{\prime}$ and $5^{\prime}$ GGACAGACTGACTTTATGGG-3 ${ }^{\prime}$; OPG, $5^{\prime}$-AGACCG TGAAACAGGAGTG- $3^{\prime}$ and $5^{\prime}$-ACCTGAGAAGAACC CATCC- $3^{\prime}$; and CTSK, $5^{\prime}$-GAAGAAGACTCACCAGAAG CAG- $3^{\prime}$ and $5^{\prime}$-TCCAGGTTATGGGCAGAGATT- ${ }^{\prime}$. The relative gene expression was calculated using the following equation: $\Delta \mathrm{Ct}=\mathrm{Ct}$ (test genes) $-\mathrm{Ct}(\mathrm{GAPDH})$; fold change $=2^{(-\Delta \mathrm{Ct})}$.

2.11. Statistical Analysis. Statistical analysis was conducted using GraphPad Prism version 6.02 software program for Windows (GraphPad, CA, USA). The Student $t$-test was used for comparison between groups. All tests were twosided, and $P$ values $<0.05$ were considered to be statistically significant.

\section{Results}

3.1. TEL Construction. SEM scanning was used to observe the surface morphology of the scaffold. We found that there were enormous micropores and irregular lamellar structures distributed on the surface of $\mathrm{nHA} / \mathrm{COL}$ scaffolds (Figure 1(a)). MSC formed finger-like filopodia and tightly adhered to the lamellar structure of scaffolds (Figure 1(b)). The Live/Dead staining showed that almost all the MSC survived in the nHA/COL scaffold, and the cells tightly adhered to the lamellar structure of the scaffold (Figure 1(c)). The scaffold has blue autofluorescence (Figure 1(c)).

3.2. Micro-CT Examination. In the control group, the newborn laminae gradually grew from bilateral vertebral pedicles to the middle and formed complete artificial laminae at the 14th week, and the scaffold degradation and trabecular bone formation proceeded orderly and alternately. In the $\beta$ catenin inhibition group, the scaffold degradation rate was significantly higher than that of the control group, and bone formation rate was significantly lower than that of the control group. In addition, its scaffold degradation rate was dominant over the bone formation, almost all the scaffold degraded at the 10th week, the newborn laminae grew slowly after the 10th week, and no complete artificial laminae were formed at the 14th week (Figure 2).

3.3. Goldner's Trichrome Staining. New bone formation was evaluated by Goldner's trichrome staining. Bone and bonelike tissues are presented as a substructure visualized in green. In the control group, the new trabecular structure formed and bone mineral deposited at the 10th week, and premature bone structure formed at the 14th week. In the $\beta$-catenin inhibition group, the scaffold degraded completely at the 10th week, the artificial laminae stopped growing, and there was still a lamina defect with the size of $400-600 \mu \mathrm{m}$ at both the 10th and $14^{\text {th }}$ weeks (Figure 3 ).

3.4. Scaffold Degradation and Bone Formation. The scaffold degradation rate was analyzed by the $3 \mathrm{D}$ reconstruction images. The scaffold degradation rate in the control group was significantly lower than that of the $\beta$-catenin inhibition group (Figure 4). In the $\beta$-catenin inhibition group, almost all the scaffold degraded at the 6th and 10th weeks (Figure 5(a)). The HE staining also showed that the scaffold degraded completely at the 10th week in the $\beta$-catenin inhibition group (Figure 5(b)). TRAP staining showed that the osteoblast numbers in the $\beta$-catenin inhibition group were significantly higher than that of the control group (Figure 5(c)). The expression levels of OPG in the control group were statistically higher than those in the $\beta$-catenin inhibition group at the 10th week, while the RANKL expression showed no statistical difference at the 10th week (Figures 5(c)-5(g)) The mRNA expression levels of CTSK in the $\beta$-catenin inhibition group were also statistically higher than those in the control group at both the 6th and 10th weeks (Figure 5(h)) The newborn laminae were also confirmed by IHC staining of OCN at the 14th week (Figure 6).

\section{Discussion}

In this study, we investigated the role of $\mathrm{Wnt} / \beta$-catenin signaling pathway in the balance of scaffold degradation and bone formation during vertebral lamina reconstruction. We found that, with the inhibition of $\beta$-catenin, the newborn laminae increased the expression levels of osteoclastic markers and the number of osteoclasts, thereby promoting the scaffold degradation and deferring the lamina formation.

Previous studies showed that the OPG-RANK-RANKL system plays the principal role in determining the balance between bone resorption and bone formation [22, 23]. Osteoblastic cell lineages can secrete RANKL, which binds to the RANK receptor on the osteoclast or osteoclast progenitor cells and activates the transcription of osteoclastic genes $[22,23]$. Osteoblastic cell lineages can also secrete OPG, which can competitively inhibit the binding between RANKL and RANK, thereby inhibiting the formation and differentiation of osteoclasts $[22,23]$. $\beta$-Catenin can induce the expression of osteoclast inhibitor OPG and regulate osteoclast differentiation, affecting bone resorption ultimately [24]. In this study, we found that the expression levels of OPG in the control group were statistically higher than those in the $\beta$-catenin inhibition group at all time points, while the expression levels of RANKL in the control group were only statistically lower than those in the $\beta$-catenin inhibition group at 6 th week. The regulatory function of $\mathrm{Wnt} / \beta$ catenin signaling pathway in the osteoclast differentiation was mainly played by promoting the expression of OPG.

The coupling balance between scaffold degradation and bone formation is requisite for bone reconstruction [25-27]. The dominance of scaffold degradation inhibits the migration and mineralization of osteoblasts, while the constrained degradation of scaffolds would squeeze the space for osteoblast proliferation [28]. In this study, the scaffold degradation rate in the $\beta$-catenin inhibition group was dominant over bone formation rate. Almost all the scaffold degraded at the $6^{\text {th }}$ week, and the bone formation retarded thereafter and could not form complete artificial laminae at the 14th week. In the control group, scaffold degradation and bone formation maintained synchronous balance and realized artificial lamina reconstruction at the 

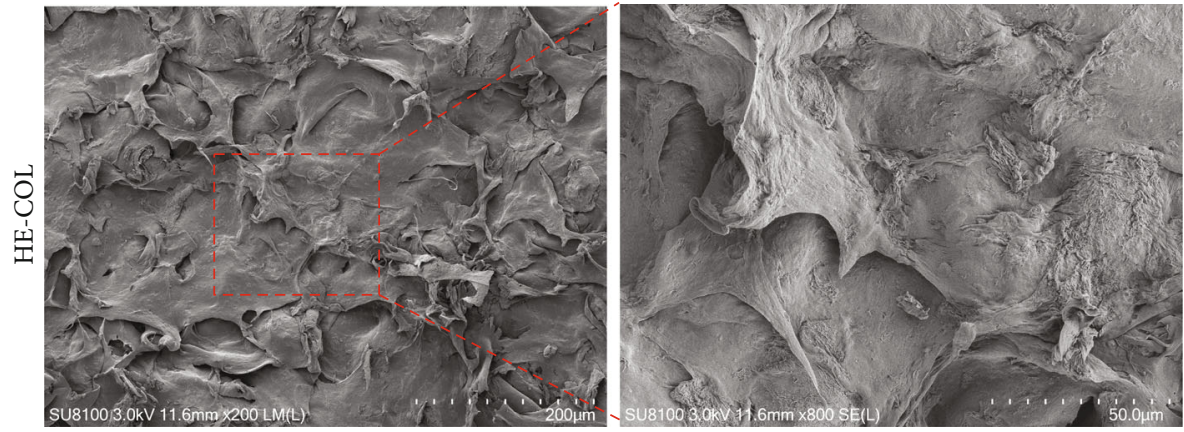

(a)
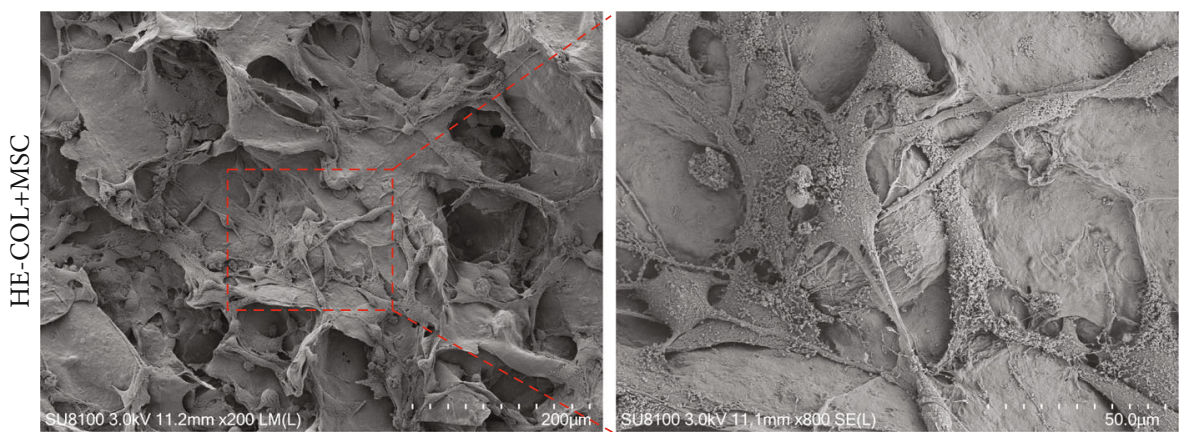

(b)


Auto-fluroscence

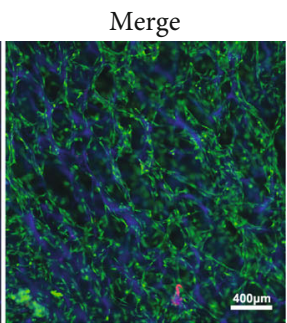

(c)

FIgure 1: (a) SEM images of nHA/COL scaffolds; (b) SEM images of tissue-engineered laminae (TEL); (c) Live/Dead staining of TEL.
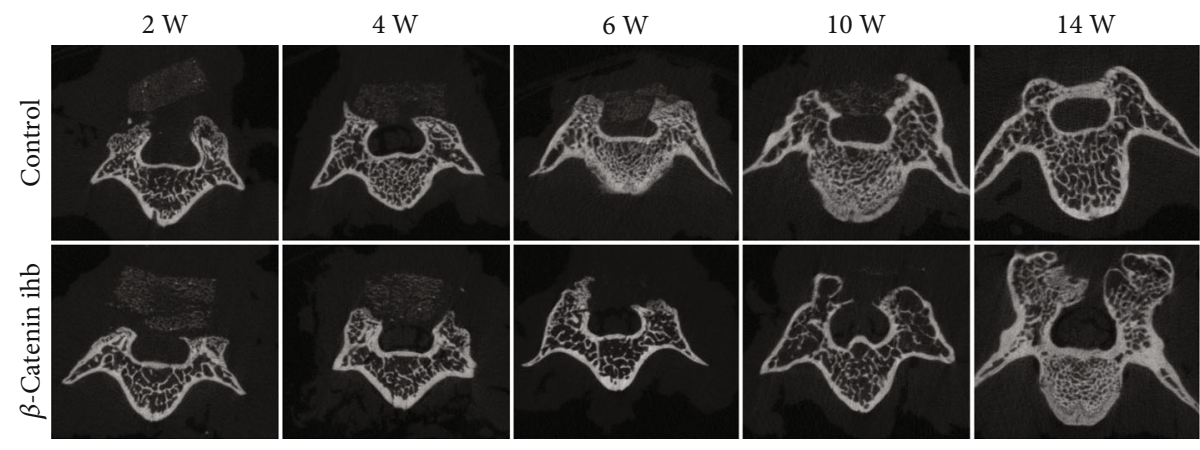

FIgURE 2: Micro-CT scanning of TEL in the control and $\beta$-catenin inhibition groups at the 2nd, 4th, 6th, 10th, and 14th weeks.

14th week. It demonstrated that $\beta$-catenin inhibition could expedite the degradation of nHE/COL scaffolds and break down the normal balance between scaffold degradation and bone formation, which was detrimental to the reconstruction of artificial vertebral laminae.

Scaffolding biomaterials, such as natural collagen, synthetic polymers, ceramics, inorganic biomaterials, and their hybrid combinations, have been widely used for bone tissue engineering, due to their outstanding biomechanical, osteoconductive, and biodegradable properties [29]. Bone regeneration is closely associated with $\mathrm{Wnt} / \beta$-catenin signaling pathway [18]. Many biomaterials, such as titanium with Ti-Nano, laponite-guanidinylated chitosan hydrogels, intrafibrillar mineralized collagen, hydroxyapatite, and 




FIGURE 3: Goldner's trichrome staining of TEL at the 2nd, 4th, 6th, 10th, and 14th weeks.

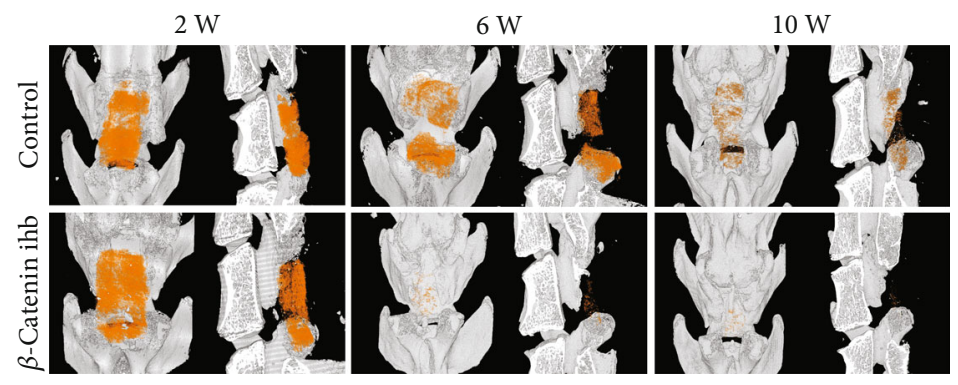

FIGURE 4: 3D reconstruction images at the 2nd, 6th, and 10th weeks; the pseudo orange color indicates the TEL.

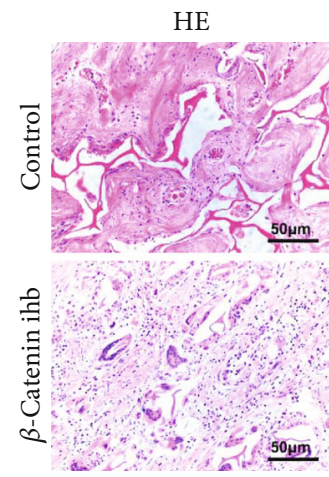

(a)



(e)



(b)

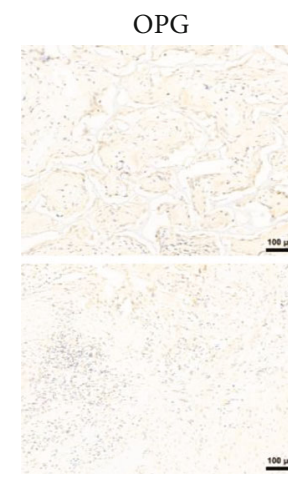

(c)

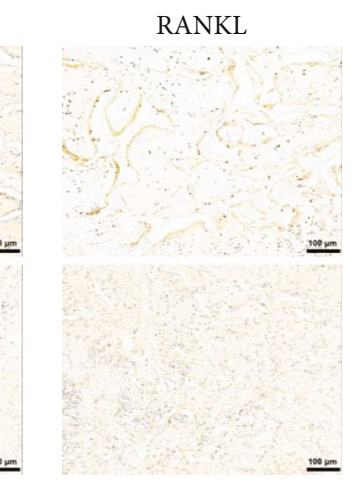

(d)



(f)



(g)



(h)

FIGURE 5: (a) HE staining of the newborn laminae at the 10th week; (b) TRAP staining of the newborn laminae at the 10th week; IHC staining of (c) OPG and (d) RANKL at the 10th week; (e) percentage of positive staining of RANKL and OPG at the 10th week; mRNA expression levels of (f) OPG, (g) RANKL, and (h) CTSK. ${ }^{*} P<0.05$ (control vs. $\beta$-catenin inhibition group at the same time point).

gold nanoparticle-loaded hydroxyapatite composites, possessed great regenerative potential because they can activate Wnt/ $\beta$-catenin signaling pathway and thereby promoting bone regeneration [30-33]. Bone progenitor stem cells, scaffolding biomaterials, and $\mathrm{Wnt} / \beta$-catenin signaling pathway must rely on each other to realize successful bone regeneration [19].

Many studies have clearly elucidated the role of $\mathrm{Wnt} / \beta$ catenin signaling pathway in promoting osteoblast proliferation, migration, and osteogenic differentiation [18, 19]. 


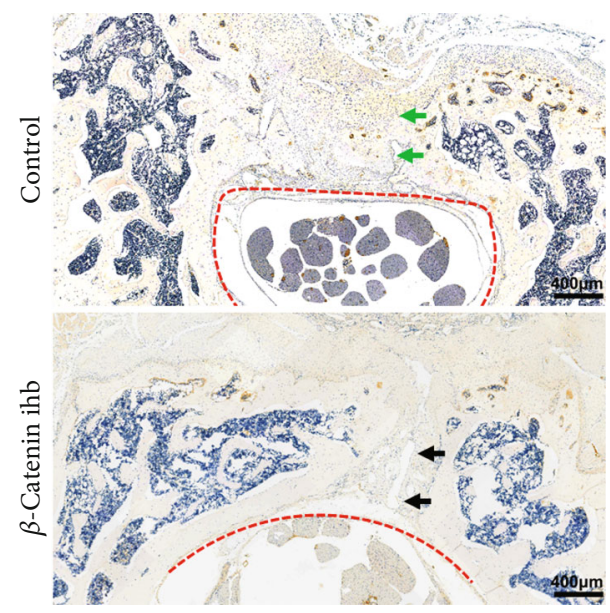

FIGURE 6: IHC staining of OCN at the 14th week. Green arrow indicates the newborn complete vertebral laminae in the control group, black arrow indicates the lamina defect in the $\beta$-catenin inhibition group, and red dish line indicates the vertebral canal.

Previous studies mostly focused on the role of biomaterials in the activation of $\mathrm{Wnt} / \beta$-catenin signaling pathway, osteogenic differentiation, and inhibition of osteoclastogenesis $[20,22,24]$. In this study, we elucidated the role of Wnt/ $\beta$-catenin signaling pathway in inhibiting osteoclast genesis and scaffold degradation and thus in maintaining the balance between scaffold degradation and bone formation. It demonstrated that $\mathrm{Wnt} / \beta$-catenin signaling pathway regulated the reconstruction of tissue-engineered bone in several different ways, which needs more attention in further study.

\section{Conclusion}

In the process of vertebral lamina reconstruction, $\mathrm{Wnt} / \beta$ catenin pathway could decrease the degradation rate of $\mathrm{nHA} /-$ COL scaffold mainly by decreasing the expression of OPG and promoting osteoclast genesis. Inhibition of $\beta$-catenin led to speedy scaffold degradation and deferred artificial lamina formation, disturbing the balance between scaffold degradation and bone formation. This study advances our understanding of the role of $\mathrm{Wnt} / \beta$-catenin pathway in bone tissue engineering.

\section{Data Availability}

The datasets used during the current study are available from the corresponding author on reasonable request.

\section{Conflicts of Interest}

The authors declare that there are no conflicts of interest regarding the publication of this paper.

\section{Authors' Contributions}

Hailong Li and Linli Li contributed equally to this work.

\section{Acknowledgments}

The study was supported by the National Natural Science Foundation of China (File No. 81672179), the Shanghai Leading Medical Talent (File No. 2019LJ08), and the Highlevel Talent Special Fund of the Minhang District of Shanghai (File No. 5-1).

\section{References}

[1] Z. Mo, R. Zhang, M. Chang, and S. Tang, "Exercise therapy versus surgery for lumbar spinal stenosis: a systematic review and meta-analysis," Pakistan Journal of Medical Sciences, vol. 34, no. 4, pp. 879-885, 2018.

[2] M. Shah, B. Kolb, E. Yilmaz, D. R. Halalmeh, and M. D. Moisi, "Comparison of lumbar laminectomy alone, lumbar laminectomy and fusion, stand-alone anterior lumbar interbody fusion, and stand-alone lateral lumbar interbody fusion for treatment of lumbar spinal stenosis: a review of the literature," Cureus, vol. 11, article e5691, 2019.

[3] J. C. le Huec, S. Seresti, S. Bourret et al., "Revision after spinal stenosis surgery," European Spine Journal, vol. 29, no. S1, pp. 22-38, 2020.

[4] A. Sebaaly, M. J. Lahoud, M. Rizkallah, G. Kreichati, and K. Kharrat, "Etiology, evaluation, and treatment of failed Back surgery syndrome," Asian Spine Journal, vol. 12, no. 3, pp. $574-585,2018$.

[5] S. Ko and T. Oh, "Comparison of bilateral decompression via unilateral laminotomy and conventional laminectomy for single-level degenerative lumbar spinal stenosis regarding low back pain, functional outcome, and quality of life - a randomized Controlled, Prospective Trial," Orthopaedic surgery and research, vol. 14, no. 1, p. 252, 2019.

[6] S. M. Park, J. Park, H. S. Jang et al., "Biportal endoscopic versus microscopic lumbar decompressive laminectomy in patients with spinal stenosis: a randomized controlled trial," The Spine Journal, vol. 20, no. 2, pp. 156-165, 2020.

[7] L. Li, X. Chen, Y. He, and Y. Dong, "Biological and mechanical factors promote the osteogenesis of rabbit artificial vertebral laminae: a comparison study," Tissue Engineering. Part A, vol. 24, no. 13-14, pp. 1082-1090, 2018.

[8] Y. Dong, X. Chen, and Y. Hong, "Tissue-engineered bone formation in vivo for artificial laminae of the vertebral arch using $\beta$-Tricalcium phosphate bioceramics seeded with mesenchymal stem cells," Spine, vol. 38, no. 21, pp. E1300-E1306, 2013.

[9] Y. Dong, X. Chen, M. Wang, and Y. Hong, "Construction of artificial laminae of the vertebral arch using bone marrow mesenchymal stem cells transplanted in collagen sponge," Spine, vol. 37, no. 8, pp. 648-653, 2012.

[10] C. Li, H. Wang, H. Liu, J. Yin, L. Cui, and Z. Chen, “The prevention effect of poly (L-glutamic acid)/chitosan on spinal epidural fibrosis and peridural adhesion in the post-laminectomy rabbit model," European Spine Journal, vol. 23, no. 11, pp. 2423-2431, 2014.

[11] J. Xu, Y. Chen, Y. Yue, J. Sun, and L. Cui, "Reconstruction of epidural fat with engineered adipose tissue from adipose derived stem cells and PLGA in the rabbit dorsal laminectomy model," Biomaterials, vol. 33, no. 29, pp. 6965-6973, 2012.

[12] X. Lei, J. Gao, F. Xing, Y. Zhang, Y. Ma, and G. Zhang, "Comparative evaluation of the physicochemical properties of nanohydroxyapatite/collagen and natural bone ceramic/collagen scaffolds and their osteogenesis-promoting effect on MC3T3- 
E1 cells," Regenerative Biomaterials, vol. 6, no. 6, pp. 361-371, 2019.

[13] X. Wang, G. Zhang, F. Qi et al., "Enhanced bone regeneration using an insulin-loaded nano-hydroxyapatite/collagen/PLGA composite scaffold," International Journal of Nanomedicine, vol. 13, pp. 117-127, 2018.

[14] Y. Xia, S. S. Peng, L. Z. Xie et al., "A novel combination of nano-scaffolds with micro-scaffolds to mimic extracellularmatrices improve osteogenesis," Journal of Biomaterials Applications, vol. 29, no. 1, pp. 59-71, 2014.

[15] N. M. Hu, Z. Chen, X. Liu et al., "Mechanical properties and in vitro bioactivity of injectable and self- setting calcium sulfate/nano-HA/collagen bone graft substitute," Journal of the Mechanical Behavior of Biomedical Materials, vol. 12, pp. 119-128, 2012.

[16] L. Li, Y. He, X. Chen, and Y. Dong, "The role of continuous cerebrospinal fluid pulsation stress in the remodeling of artificial vertebral laminae: a comparison experiment," Tissue Engineering. Part A, vol. 25, no. 3-4, pp. 203-213, 2019.

[17] L. Li, Y. He, H. Tang et al., "Cerebrospinal Fluid Pulsation Stress Promotes the Angiogenesis of Tissue- Engineered Laminae," Stem Cells International, vol. 2020, Article ID 8026362, 12 pages, 2020.

[18] P. Leucht, S. Lee, and N. Yim, "Wnt signaling and bone regeneration: can't have one without the other," Biomaterials, vol. 196, pp. 46-50, 2019.

[19] J. B. Kim, P. Leucht, K. Lam et al., "Bone regeneration is regulated by wnt signaling," Journal of Bone and Mineral Research, vol. 22, no. 12, pp. 1913-1923, 2007.

[20] M. Hadjiargyrou, F. Lombardo, S. Zhao et al., "Transcriptional Profiling of Bone Regeneration: insight into the molecular complexity of wound repair," Journal of Biological Chemistry, vol. 277, no. 33, pp. 30177-30182, 2002.

[21] N. Zhong, R. P. Gersch, and M. Hadjiargyrou, "Wnt signaling activation during bone regeneration and the role of Dishevelled in chondrocyte proliferation and differentiation," Bone, vol. 39, no. 1, pp. 5-16, 2006.

[22] L. Zhou, Y. Huang, J. Zhao, H. Yang, and F. Kuai, “Oridonin promotes osteogenesis through $\mathrm{Wnt} / \beta$-catenin pathway and inhibits RANKL-induced osteoclastogenesis in vitro," Life Sciences, vol. 262, 2020.

[23] B. Kadriu, P. W. Gold, D. A. Luckenbaugh et al., "Acute ketamine administration corrects abnormal inflammatory bone markers in major depressive disorder," Molecular Psychiatry, vol. 23, no. 7, pp. 1626-1631, 2018.

[24] I. Kramer, C. Halleux, H. Keller et al., "Osteocyte Wnt/betacatenin signaling is required for normal bone homeostasis," Molecular and Cellular Biology, vol. 30, no. 12, pp. 30713085, 2010.

[25] E. Alsberg, H. J. Kong, Y. Hirano, M. K. Smith, A. Albeiruti, and D. J. Mooney, "Regulating bone formation via controlled scaffold degradation," Journal of Dental Research, vol. 82, no. 11, pp. 903-908, 2003.

[26] Q. Shi, H. Shui, Q. Chen, and Z. Y. Li, "How does mechanical stimulus affect the coupling process of the scaffold degradation and bone formation: An in silico approach," Computers in Biology and Medicine, vol. 117, 2020.

[27] Q. Zhao, H. Tang, L. Ren, and J. Wei, "In vitro apatite mineralization, degradability, cytocompatibility and in vivo new bone formation and vascularization of bioactive scaffold of polybutylene succinate/magnesium phosphate/wheat protein ternary composite," International Journal of Nanomedicine, vol. 15, pp. 7279-7295, 2020.

[28] X. Huang, H. Xiao, and F. Xue, "Ectopic osteogenesis and scaffold biodegradation of hydroxyapatite-collagen I scaffold in a rabbit mode," Journal of Biomaterials and Tissue Engineering, vol. 8, pp. 1315-1319, 2018.

[29] S. Pina, V. P. Ribeiro, C. F. Marques et al., "Scaffolding strategies for tissue engineering and regenerative medicine applications," Materials, vol. 12, no. 11, 2019.

[30] R. Abuna, F. S. Oliveira, H. B. Lopes et al., "The Wnt/ $\beta$-catenin signaling pathway is regulated by titanium with nanotopography to induce osteoblast differentiation," Colloids and Surfaces. B, Biointerfaces, vol. 184, 2019.

[31] Z. Zhang, Z. Li, C. Zhang et al., "Biomimetic intrafibrillar mineralized collagen promotes bone regeneration via activation of the Wnt signaling pathway," International Journal of Nanomedicine, vol. 13, pp. 7503-7516, 2018.

[32] X. Zhang, J. Fan, C. S. Lee, S. Kim, C. Chen, and M. Lee, "Supramolecular hydrogels based on nanoclay and guanidine-rich chitosan: injectable and moldable osteoinductive carriers," ACS Applied Materials \& Interfaces, vol. 12, no. 14, pp. 1608816096, 2020.

[33] H. Liang, X. Xu, X. Feng et al., "Gold nanoparticles-loaded hydroxyapatite composites guide osteogenic differentiation of human mesenchymal stem cells through $\mathrm{Wnt} / \beta$-catenin signaling pathway," International Journal of Nanomedicine, vol. 14, pp. 6151-6163, 2019. 